\title{
THE
}

\section{Excitation Spectrum and Thermodynamic Properties of the Ising- Heisenberg Linear Ferromagnet}

\author{
J. D. Johnson \\ Jill C. Bonner \\ University of Rhode Island
}

Follow this and additional works at: https://digitalcommons.uri.edu/phys_facpubs

Terms of Use

All rights reserved under copyright.

\section{Citation/Publisher Attribution}

Johnson, J. D., \& Bonner, J. C. (1980). Excitation spectrum and thermodynamic properties of the IsingHeisenberg linear ferromagnet. Phys. Rev. Lett. 44(9), 616-619. doi: 10.1103/PhysRevLett.44.616 Available at: http://dx.doi.org/10.1103/PhysRevLett.44.616 


\title{
Excitation Spectrum and Thermodynamic Properties of the Ising-Heisenberg Linear Ferromagnet
}

\author{
J. D. Johnson \\ Theoretical Division, Los Alamos Scientific Laboratory, Los Alamos, New Mexico 87545 \\ and \\ J. C. Bonner \\ Physics Department, University of Rhode Island, Kingston, Rhode Island 02881
}

(Received 9 November 1979)

\begin{abstract}
New analytic results are presented for the low- $T$ thermodynamics of the IsingHeisenberg linear ferromagnetic in a magnetic field $H_{0}$. For small $H_{0}$ the thermodynamic functions show unexpected and interesting structure as a function of $H_{0}$ and the anisotropy $\Delta$. The thermal and magnetic energy gaps have singularities, not necessarily at the same $\Delta-H_{0}$ location, as changes occur in the type of excitation dominating the low- $T$ behavior. The results may relate to quantum solitons in the linear ferromagnet.
\end{abstract}

There has been a renewed interest in exact solutions of nontrivial, quantum-mechanical, onedimensional models. ${ }^{1}$ For example, exact and fairly complete solutions are now available for the one-dimensional (1D), $\delta$-function potential, Fermi- and Bose-gas models, ${ }^{2}$ the linear Hubbard model of a metal-insulator transition, ${ }^{3}$ and the linear, spin- $\frac{1}{2}$, Ising-Heisenberg $X Y$ continuum model. ${ }^{4}$ Exact solutions for a continuum electron gas ${ }^{5}$ and an electron gas on a lattice ${ }^{6}$ are known. These are relevant to the important field of 1D organic conductors. ${ }^{7}$ Models for organic charge-transfer salts can be mapped into a quantum magnetic chain ${ }^{8}$ which in the antiferromag- netic limit corresponds to the Hubbard dimer gas. ${ }^{9}$ The exact solutions of the 1D quantummechanical sine-Gordon and related equations (solitons) have been extensively applied to chargedensity waves in 1D conductors. ${ }^{6,10}$ Very recently the Bethe's Ansatz techniques have been used to solve the massive Thirring model. ${ }^{11,12}$ Fadeev's review presents a unified approach to all the models discussed above. ${ }^{12}$ Sutherland gives an overview of the quantum soliton concept and its connections to Bethe's Ansatz. ${ }^{13}$

In this Letter we present new, unanticipated, and interesting exact results for the $1 \mathrm{D}$, spin- $-\frac{1}{2}$, ferromagnetic, Ising-Heisenberg model. The Hamiltonian $^{14}$ is

$$
H=-\sum_{i=1}^{N}\left[S_{i}{ }^{x} S_{i+1}{ }^{x}+S_{i}{ }^{y} S_{i+1}{ }^{y}+\Delta\left(S_{i}{ }^{z}{S_{i+1}}^{z}-\frac{1}{4}\right)\right]-H_{0} \sum_{i=1}^{N} S_{i}{ }^{z} .
$$

The $S$ 's are $\frac{1}{2}$ the respective Pauli matrices, and there are periodic boundary conditions on the system. $\Delta \geqslant 1$ except, if $H_{0}$ is in a small, order $T^{0}$, neighborhood of zero, we restrict $\Delta>1$. ( $T$ is temperature.) We set Boltzmann's constant to 1 throughout the body of this paper.

This system was first studied ${ }^{15}$ in the 1930 's; a formalism for the thermodynamics was derived by Gaudin much later. ${ }^{16}$ In Gaudin's work assumptions were made which are difficult to verify directly. In this and previous work ${ }^{17}$ we have made comparisons of the predictions of Gaudin's formalism to numerical results on finite systems. ${ }^{18}$ All comparisons are favorable thus enhancing our faith in the assumptions contained in the thermodynamic formalism.

We have performed low-temperature expansions of Gaudin's formalism to derive all of our results. We will not present this approach in this paper, however, since it is a fairly long and detailed derivation. We will give a "physical" argument for the results which shows the connection between the lowtemperature thermodynamics and the excitations of the system.

It is known that the zero-temperature dispersion curves for this system ${ }^{17}$ are given by

$$
E_{n}(P)=n H_{0}+\sinh \Phi(\cosh n \Phi-\cos P) / \sinh n \Phi,
$$

where $\Delta=\cosh \Phi, 0 \leqslant P \leqslant 2 \pi$, and $n=1,2, \ldots$. The $n=1$ excitations and linear combination of the $n=1$ excitations are spin waves, and the higher $-n$ excitations are bound states of spin waves. The $P$ 's are distributed uniformly between 0 and $2 \pi$ and, for a given $n$, obey a Fermi-like exclusion principle. 
The energies of the first excited states are $E(q)=H_{0}+\Delta-\cos q$. There are $N$ such states with $q$ $=2 \pi m / N, 0 \leqslant q \leqslant 2 \pi$. The states we first sum to derive the partition function are these $N$ states, the $\frac{1}{2} N(N-1)$ states with energies $E\left(q_{1}\right)+E\left(q_{2}\right), \ldots$, the $N ! /[l !(N-l)]$ states with energies $E\left(q_{1}\right)+E\left(q_{2}\right)$ $+\ldots+E\left(q_{l}\right)$, etc. These are all the spin-wave excitations, and they provide a contribution ${ }^{17}$ to $F(T, \sigma)$ of

$$
F(T, \sigma)=\sigma H_{0}-(T / 2 \pi) \int_{0}^{2 \pi} d q \exp \left[-\left(\Delta+H_{0}-\cos q\right) / T\right],
$$

where $\sigma$ is the magnetization per spin.

For $H_{0}$ far enough away from zero, this is all we need to obtain the low-temperature thermoydnamics to exponential accuracy in $T$. However, for small $H_{0}$ other excitations, the high-lying bound states, can dominate. For large $n E_{n}(P) \sim n H_{0}+\sinh \Phi$; note that the $E_{n}(P)$ are independent of $P$ and, according$1 y$, are effectively just the energies of a 1D Ising model with exchange constant $J=\sinh \Phi$. Therefore, we add to Eq. (3) the Ising free energy for this $J .^{19}$ We obtain, after some simplification for low $T$ of the Ising-model result,

$$
F(T, \sigma)-\sigma H_{0}=-\left\{H_{0}^{2} / 4+T^{2} \exp \left[-\left(\Delta^{2}-1\right)^{1 / 2} / T\right]\right\}^{1 / 2}-(T / 2 \pi) \int_{0}^{2 \pi} d q \exp \left[-\left(\Delta+H_{0}-\cos q\right) / T\right]+E .
$$

This is our basic result and is the same result as obtained by the low- $T$ expansion of the Gaudin formalism. It is valid for low $T, O(T)>H_{0} \geqslant 0$ and $\Delta>1$. The correction, $E$, is exponentially higher order in $T$ than the larger of the two terms on the right-hand side of Eq. (4) even after taking an arbitrary number of $T$ derivatives or up to and including two $H_{0}$ derivatives. \{Note that this means, in particular, that if one expands the square root for $H_{0}$ exponentially larger or smaller than exp $\left[-\left(\Delta^{2}\right.\right.$ $\left.-1)^{1 / 2} / 2 T\right]$, one should retain two terms in the expansion. Both terms are significant, and $E$ is exponentially higher order than the integral or the second term of the square-root expansion, whichever is larger. ${ }^{20}$

We now discuss the detailed behavior of Eq. (4) in terms of the susceptibility $\chi \equiv-\left[\partial^{2}\left(F-\sigma H_{0}\right) / \partial H_{0}^{2}\right]_{T}$ and specific heat $C_{H} \equiv-T\left[\partial^{2}\left(F-\sigma H_{0}\right) / \partial T^{2}\right]_{H_{0}}$. We find from Eq. (4)

$$
\begin{aligned}
& \chi=T^{2} \exp \left[-\left(\Delta^{2}-1\right)^{1 / 2} / T\right]\left(4\left\{H_{0}^{2} / 4+T^{2} \exp \left[-\left(\Delta^{2}-1\right)^{1 / 2} / T\right]\right\}^{3 / 2}\right)^{-1} \\
&+(2 \pi T)^{-1} \int_{0}^{2 \pi} d q \exp \left[-\left(\Delta+H_{0}-\cos q\right) / T\right]+E_{\mathrm{\chi}} .
\end{aligned}
$$

$E_{\mathrm{X}}$ is exponentially higher order in $T$ than the larger of the first two terms. If we asymptotically expand the integral,

$$
\begin{aligned}
\chi=T^{2} \exp \left[-\left(\Delta^{2}-1\right)^{1 / 2} / T\right]\left(4 \left\{H_{0}{ }^{2} / 4+T^{2}\right.\right. & \left.\left.\exp \left[-\left(\Delta^{2}-1\right)^{1 / 2} / T\right]\right\}^{3 / 2}\right)^{-1} \\
& +(2 \pi T)^{-1 / 2} \exp \left[-\left(\Delta+H_{0}-1\right) / T\right]+E_{\chi}{ }^{\prime} .
\end{aligned}
$$

$E_{\chi}{ }^{\prime}$ is exponentially higher order than the first term of Eq. (6a) or $O(T)$ higher order than the second term, whichever is larger. Similarly, for $C_{H}$, we obtain

$$
\begin{aligned}
C_{H}=\left(\Delta^{2}-1\right) \exp \left[-\left(\Delta^{2}-1\right)^{1 / 2} / T\right]\left\{H_{0}{ }^{2} / 2+T^{2} \exp \left[-\left(\Delta^{2}-1\right)^{1 / 2} / T\right]\right\} \\
\quad \times\left(4 T\left\{H_{0}^{2} / 4+T^{2} \exp \left[-\left(\Delta^{2}-1\right)^{1 / 2} / T\right]\right\}^{3 / 2}\right)^{-1}+\left(2 \pi T^{3}\right)^{-1 / 2}\left(\Delta+H_{0}-1\right)^{2} \exp \left[-\left(\Delta+H_{0}-1\right) / T\right]+E_{c} .
\end{aligned}
$$

$E_{c}$ is $O(T)$ higher order than the larger of the first two terms. The first terms of both $\chi$ and $C_{H}$ are bound-state contributions and the second terms are spin-wave contributions.

For $\chi$ we redefine variables to $H_{0}=e^{\alpha / T}$; Fig. 1 illustrates the following discussion. For $\alpha>\alpha_{b}$ $=\frac{1}{3}\left[\Delta-1-\left(\Delta^{2}-1\right)^{1 / 2}\right]$ the spin-wave term dominates the bound-state term. For $\alpha<\alpha_{b}$ the boundstate term dominates. Obvious simplifications can be made to either Eq. (5) or (6a) by dropping appropriate terms in these cases. The boundstate region subdivides into $\alpha<\alpha_{c}=-\left(\Delta^{2}-1\right)^{1 / 2} / 2$ and $\alpha>\alpha_{c}$. For $\alpha<\alpha_{c}$, Eq. (5) simplifies to

$$
\chi=(4 T)^{-1} \exp \left[\left(\Delta^{2}-1\right)^{1 / 2} /(2 T)\right] \text {. }
$$

For $\alpha_{b}>\alpha>\alpha_{c}$,

$$
\chi=2 T^{2} H_{0}^{-3} \exp \left[-\left(\Delta^{2}-1\right)^{1 / 2} / T\right] .
$$

Corrections to both these equations are exponentially higher order in $T$. We thus have three separate regions for $\chi$ with different exponential behavior in each.

$C_{H}$ is somewhat different from $\chi$ and is illustrat- 


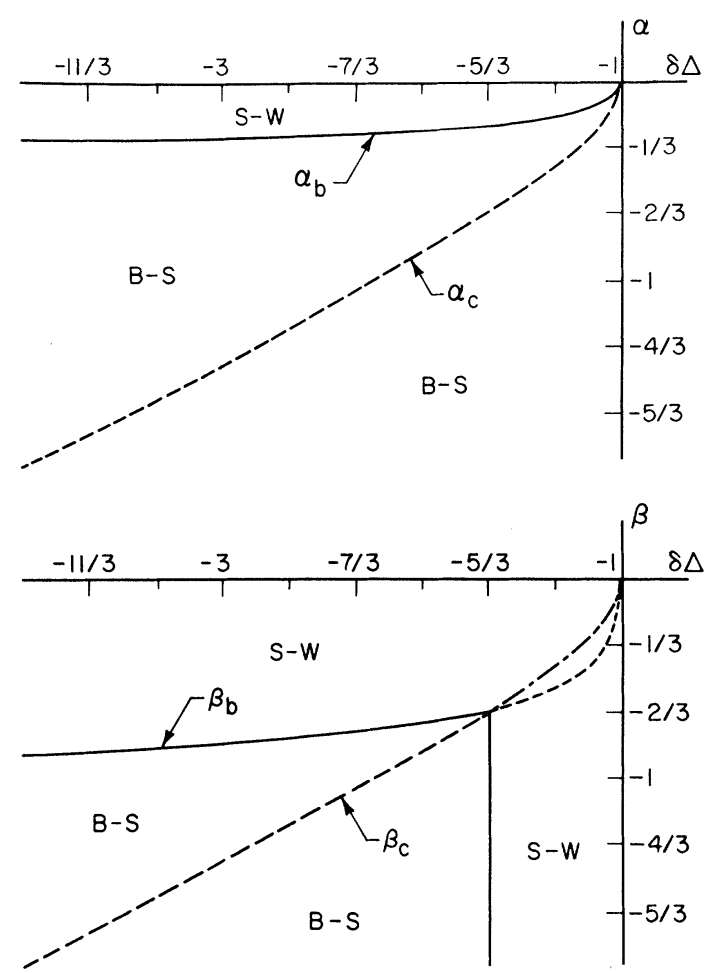

FIG. 1. The top portion presents the various regions for $\chi$, the susceptibility. The bottom portion illustrates the character of $C_{H}$, the specific heat. In both cases S-W labels the spin-wave regions, B-S labels the boundstate regions, and $\delta=-1$.

ed in Fig. 1. We define $H_{0}=e^{\beta / T}$. Then for $\beta>\beta_{b}$ $=\Delta-1-\left(\Delta^{2}-1\right)^{1 / 2}=3 \alpha_{b}$ and also for $\frac{5}{3}>\Delta>1$, all $\beta$, the spin waves dominate. One can then drop the first term in Eq. (6b). For $\beta<\beta_{b}, \Delta>\frac{5}{3}$, the bound states dominate and one can drop the second term. The bound-state region again subdivides into $\beta<\beta_{c}=-\left(\Delta^{2}-1\right)^{1 / 2} / 2=\alpha_{c}$ and $\beta>\beta_{c}$. For $\beta>\beta_{c}$ with $\Delta>\frac{5}{3}$,

$$
C_{H}=\left(T H_{0}\right)^{-1}\left(\Delta^{2}-1\right) \exp \left[-\left(\Delta^{2}-1\right)^{1 / 2} / T\right]
$$

and, for $\beta<\beta_{c}$ with $\Delta>\frac{5}{3}$,

$$
C_{H}=\left(4 T^{2}\right)^{-1}\left(\Delta^{2}-1\right) \exp \left[-\left(\Delta^{2}-1\right)^{1 / 2} /(2 T)\right] .
$$

The corrections are $O(T)$ and exponentially higher order for Eqs. (8a) and (8b), respectively. Again one has three separate regions with different exponential behavior in each (as for $\chi$ ), but the details are different from $x$.

To emphasize this difference between $\chi$ and $C_{H}$, look at $H_{0}=0$. For $H_{0}=0 \chi$ is given by Eq. (7a) to exponential accuracy for all $\Delta>1$. $C_{H}$, however, is given by Eq. (8b) for $\Delta>\frac{5}{3}$ and by

$$
C_{H}=\left(2 \pi T^{3}\right)^{-1 / 2}(\Delta-1)^{2} e^{-(\Delta-1) / T}
$$

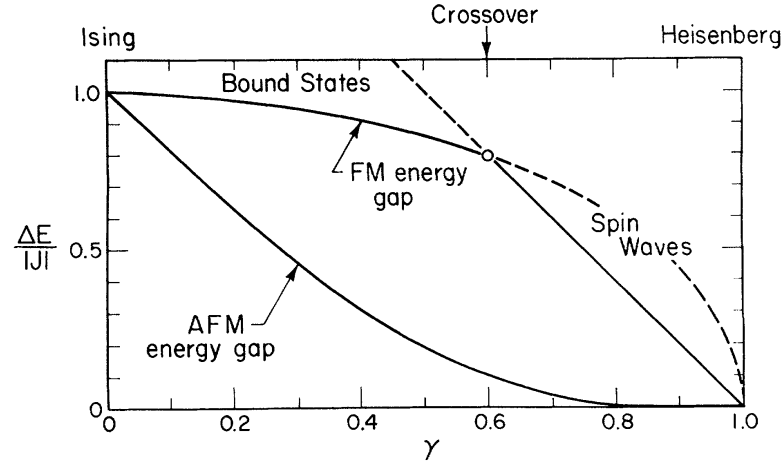

FIG. 2. We plot the effective energy gap for $C_{H}$. Shown are the gaps for the ferromagnet and antiferromagnet. The ferromagnet curve illustrates the singularity (kink) described in the text. The antiferromagnet gap is given by a single expression over the whole anisotropy range.

for $\frac{5}{3}>\Delta>1$. Corrections to Eq. (9) are $O(T)$ higher order. Thus $\chi$ has a single effective gap for all $\Delta$ at $H_{0}=0$ while $C_{H}$ has two effective gaps with a crossover between the two at $\Delta=\frac{5}{3} .{ }^{21}$ The bound states dominate for large $\Delta$ while the spin waves dominate for small $\Delta$. This is shown in Fig. 2, where the notation is that of Ref. 14 .

It would be of considerable interest to investigate these crossover effects experimentally. Farinfrared studies like those of Torrance and Tinkham ${ }^{22}$ on $\mathrm{CoCl}_{2} \cdot 2 \mathrm{H}_{2} \mathrm{O}$ might be performed on the Ising-like linear ferromagnet cobalt chloride dipyridine $\left(\mathrm{CoCl}_{2} \cdot 2 \mathrm{NC}_{5} \mathrm{H}_{5}\right)$. The very recent discovery of a family of good Heisenberg-like ferromagnets ${ }^{23}$ offers the possibility of studies by neutrons or other means of the more isotropic region. Finally, we note that an understanding of the excitations of the linear ferromagnet may be important for the quantum soliton problem. ${ }^{24}$

This work was supported in part by the U. S. Department of Energy, Contract No. W-7405ENG-36, in part by the National Science Foundation, Grant No. DMR77-24136, and in part by NATO. One of us (J.C.B.) is a Fellow of the Bunting Institute, Radcliffe College, Cambridge, Mass. 02138.

${ }^{1}$ J. C. Bonner, J. Appl. Phys. 49, 1299 (1978).

${ }^{2}$ C. K. Lai, Phys. Rev. A 8 , 2567 (1973); M. Takahashi, Prog. Theor. Phys. 46, 1388 (1971); H. G. Vaidya and C. A. Tracy, Phys. Rev。Lett. $\underline{42}, 3$ (1979), and $\underline{43}, 1540$ (1979).

${ }^{3}$ M. Takahashi, Prog. Theor. Phys. $\underline{43}, 1619$ (1970), and 52, 103 (1974).

${ }^{4}$ A. Luther and I. Peschel, Phys. Rev. B 12, 3908 (1975). 


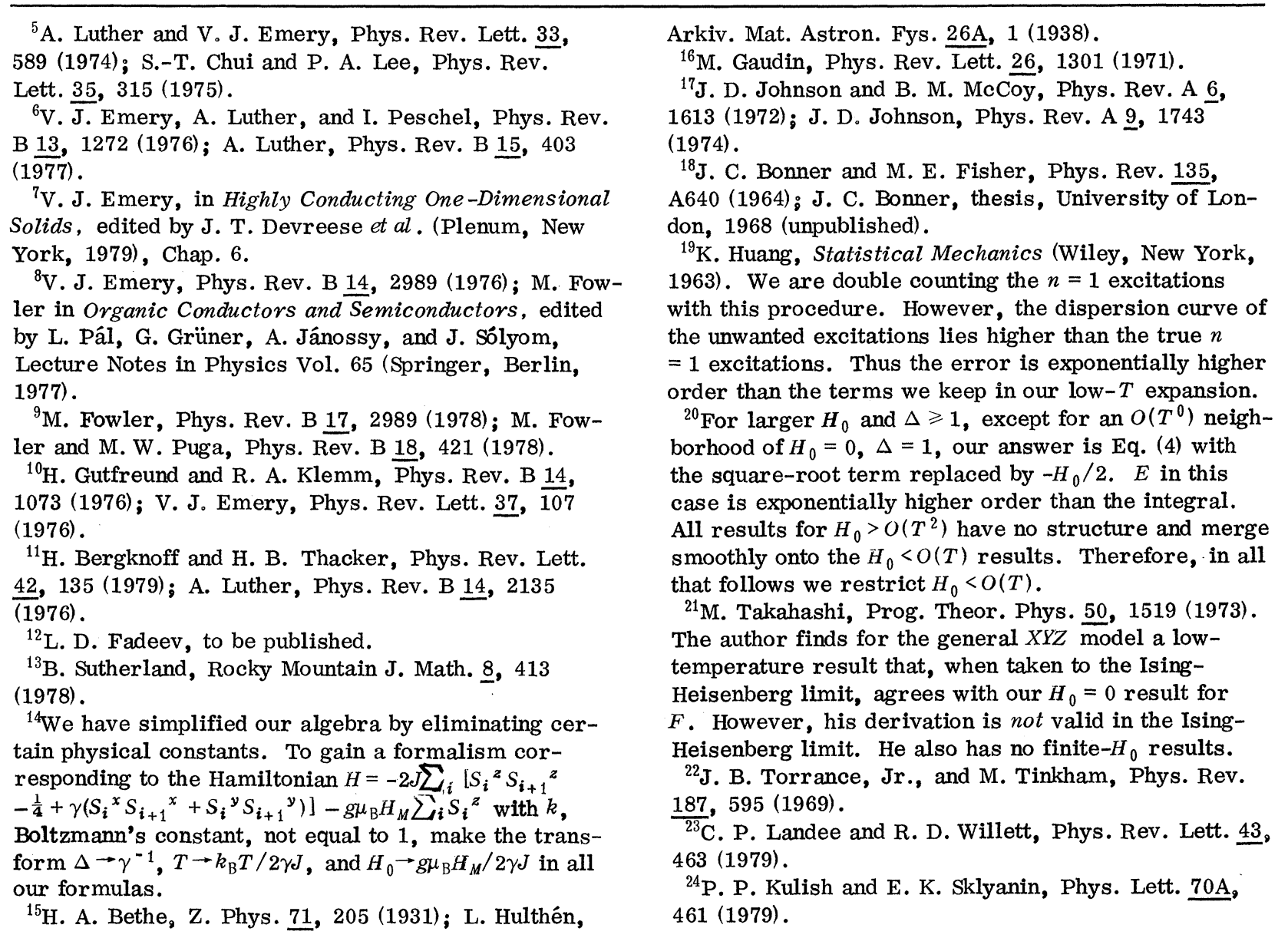

ERRATA

NEW APPROACH TO PERTURBATION THEORY. Y. Aharonov and C. K. Au [Phys. Rev. Lett. $\underline{42}$, 1582 (1979), and 43, 176(E) (1979)].

Equations (12) and (40) in this paper should read as follows:

$$
\begin{aligned}
& -\frac{1}{2}\left(g^{2}-g^{\prime}\right) e^{-G}=\left(E-V_{0}-\lambda V_{1}\right) e^{-G} \\
& F_{i}(x) \equiv 2 \alpha_{i-1}\left[g_{1}+g_{0} \alpha_{1} /\left(x-\alpha_{0}\right)\right] \exp \left(-2 G_{0}\right) \\
& \quad+\sum_{m=2}^{i-1} \alpha_{i-m}\left[\sum_{j=0}^{m} g_{j} g_{m-j}-g_{m}{ }^{\prime}+2 E_{m}\right]\left(x-\alpha_{0}\right) \exp \left(-2 G_{0}\right)-\sum_{j=1}^{i=1} g_{j} g_{i-j}\left(x-\alpha_{0}\right)^{2} \exp \left(-2 G_{0}\right) .
\end{aligned}
$$

